\title{
L'IDENTITÉ: DE LA SOCIOLOGIE AUX SCIENCES SOCIALES
}

\author{
LA IDENTIDAD: DE LA SOCIOLOGÍA A LAS CIENCIAS SOCIALES \\ IDENTITY: FROM SOCIOLOGY TO SOCIAL SCIENCES
}

\author{
Yasmine Alaoui \\ ESCA Ecole de Management, Casablanca. Marruecos/Morocco \\ alaouiyasmine1@gmail.com \\ Mostafa Abakouy \\ Université Abdelmalek Essaâdi, Tánger. Marruecos/Morocco \\ mabakouyable@gmail.com
}

Recibido/Received: 30/11/2016

Modificado/Modified: 03/06/2017

Aceptado/Accepted: 05/10/2017

\section{RÉSUMÉ}

Cet article a pour principal objectif d'explorer la notion de l'identité sous différents angles: ethnographie, sociologie, psychologie, psychanalyse anthropologie, géographie et urbanisme. Le dessein de cet article n'est pas de recenser les aspects de l'identité de manière exhaustive, mais de faire un tour d'horizon des principaux domaines qui ont traité l'identité territoriale et proposer une typologie qui va de l'identité personnelle à l'identité territoriale en passant par l'individuelle et la collective. A la genèse de ce papier des questionnements: Que veut-on dire par l'identité ? Quelle est sa relation avec le territoire et est ce qu'elle peut être un levier d'attractivité territoriale réussie ?, la volonté de développer cette notion a été pimentée par le sentiment d'urgence manifesté par la majorité des territoires dans la quête continue de la valorisation de leur identité souffrante de la "maladie" du siècle: la mondialisation.

\section{MOTS-CLÉS}

Identité; territoire; identité territoriale; attractivité.

\section{SOMMAIRE}

1. Introduction. 2. La genèse du concept de l'identité. 3. Vers une typologie de l'identité territoriale. 4. L'identité territoriale, pierre angulaire de l'attractivité. 5. Conclusion. Bibliographie

\section{RESUMEN}

Este artículo tiene como principal objetivo explorar el concepto de identidad desde distintos angulos: etnografía, sociología, psicología, antropología, geografía y urbanismo. No se trata de contabilizar los aspectos de la identidad de manera exhaustiva sino hacer un repaso general de los principales ámbitos que tratan la identidad territorial y proponer una tipología que va desde la identidad personal hasta la identidad territorial pasando por la individual y la colectiva. Algunas cuestiones a las que se quieren dar respuesta desde los inicios: ¿Qué se quiere cuando se habla de identidad? ¿Qué relación existe entre la identidad y el territorio? ¿Puede transformarse el sentimiento de identidad en una palanca de poder territorial exitoso? El objetivo de desarrollar este concepto está fundamentado en el sentimiento urgentemente manifestado por la mayoría de los territorios en la búsqueda continua por dar valor a su identidad dañada por la "enfermedad" del siglo: la globalización. 


\title{
PALABRAS CLAVE
}

Identidad; territorio; identidad territorial; atracción; universalización.

\section{SUMARIO}

1. Introducción. 2. Génesis del concepto de identidad. 3. Hacia una tipología de la identidad territorial. 4. La identidad territorial, piedra angular de la atracción. 5. Conclusión. Bibliografía.

\begin{abstract}
The main objective of this paper is to explore the notion of identity from different angles: ethnography, sociology, psychology, psychoanalysis, anthropology, geography and urbanism. The aim of this article is not to list the aspects of identity exhaustively, but to give an overview of the main areas that have dealt with territorial identity and propose a typology ranging from personal identity to the territorial identity through the individual and the collective. At the genesis of this paper many questions: What does one mean by identity? What is the relation between identity and territory and can it be a lever of territorial attractiveness? The desire to develop this notion has been spiced up by the sense of urgency manifested by the majority of territories in the continuous search for the valorization of their suffering identity of the"disease" of the century: globalization.
\end{abstract}

\section{KEYWORDS}

Identity; territory; territorial identity; attractiveness; globalization.

\section{CONTENTS}

1. Introduction. 2. The genesis of the concept of identity. 3. To a typology of territorial identity. 4 . Territorial identity, the cornerstone of attractiveness. 5. Conclusion. References.

\section{INTRODUCTION}

Le concept d'identité est particulièrement complexe, en effet, sa nature multidisciplinaire rend sa définition malaisée. Le but de ce papier n'est pas de faire état de l'art de manière intégrale de l'ensemble des travaux et disciplines traitant de l'identité, mais nous tenterons d'éclairer le concept et de mettre la lumière sur son évolution.

A première vue, l'identité apparaît comme une notion simple et claire. Cependant, lorsqu'on est amené à l'étudier, elle s'avère être d'une grande difficulté. Elle peut même, dans un certain sens, paraître contradictoire car elle possède des significations parfois opposées.

La notion d'identité n'est pas neutre, ni socialement ni politiquement. La nature paradoxale du terme dans des domaines multiples et différents fait que l'étude soit apparemment ambiguë, elle est assez timorée et très utilisé en sciences sociales pour dénoter plusieurs phénomènes. On peut ainsi noter de multiples catégories d'identités: individuelle, collective, sociale, personnelle, etc. Cette notion est à la fois polysémique et transdisciplinaire; elle est donc souvent source d'embrouillement.

Partant du principe que l'identité est une mine de confusions, le fait de parler d'identité du territoire semble être redondant car les identités sont presque toujours territorialisées (Di Méo, 2006).

Il n'est pas possible de faire ici un examen complet du concept. Nous nous satisferons de quelques visions pour mettre en relief ses multiples facettes et mettre en valeur l'orientation que nous voulons analyser dans notre démarche. 


\subsection{Objectifs et méthodologie}

Nous nous intéressons dans cet article à étudier la notion d'identité du territoire, pour ensuite la mettre en rapport avec la notion de la sociologie.

L'intérêt majeur que nous portons au concept de l'identité est centré sur le fait qu'il soit un "concept opératoire pour la recherche en sciences sociales" (Di Méo, 2006) et en tant que volet complémentaire de la démarche du plan stratégique d'un territoire (Noisette \& Vallerugo, 1996).

Nous allons de prime à bord apporter les éléments nécessaires pour définir l'identité de manière globale puis, nous nous attacherons à déterminer sa typologie. Nous aborderons par la suite, la question de l'identité territoriale après avoir déficeler les soubassements de la notion sous l'ongle de la sociologie. Nous terminerons par l'exposition de l'identité sociale perçue d'après nos recherches comme base de l'attractivité territoriale durable.

\section{LA GENESE DU CONCEPT DE L'IDENTITÉ}

L'identité peut faire référence à des réalités et des faits multiples et différents, de ce fait, une réflexion rétroactive consistant à faire retours sur son sens étymologique s'impose.

Beaucoup sont les chercheurs qui confirment que l'identité est un concept apparu initialement dans le champ de la psychologie sociale, mais il se trouve en vérité que cette notion plonge ses racines dans des domaines distincts; l'anthropologie, la psychologie génétique, la psychanalyse, la pensée Sartrienne, on peut aussi la trouver dans le domaine mathématique dans les identités remarquables par exemple. Ou encore dans les champs administratifs, par exemple dans les papiers d'identités, qui permettent la reconnaissance des individus dans la société. Aujourd'hui, l'identité se présente comme la synthèse de tous ces courants et constitue l'une des plus grandes notions transversales des sciences humaines et sociales.

Rey (1998), a réalisé une étude étymologique dans laquelle il démontre que le terme identité est apparu au XIVe siècle dans la langue française, ses racines étymologiques proviennent du mot latin "identitas" dérivé du latin classique "ídem" qui veut dire "la même chose". Au XVIIIe siècle, le terme identité était définit comme le "caractère de deux objets de pensée identique".

D`autr part, le mot a fait référence au "permanent", il s'agit du caractère de toute chose permanente qui reste intacte pour une longue période. Plus tard en 1797, le mot s'inscrit dans le sens de la logique et s'emploie de la même façon qu' "identique", c'est-à-dire le caractère de ce qui est un. En 1840, il est utilisé comme un terme d'algèbre.

Quarante ans après, c'est le droit qui emprunte le terme et on commence à entendre parler de "plaque d'identité", à partir du XXe siècle, l'identité est entrée dans le langage judiciaire désignant les services de police charges de l'identification des personnes (Rey, 1998).

Malgré l'évolution de l'utilisation et des interprétations données à l'identité, le mot a conservé une syntaxe quasi intacte et similaire dans toutes les langues occidentales, par exemple; l'espagnol identidad, l'italien identità et la française identité. Il est à noter aussi que les langues germaniques ont également gardés la racine latine, l'anglais identity, et l'allemand Identitat.

Dans ce qui suit, nous allons essayer de faire une recension des applications et des interprétations pluridisciplinaire du concept de l'identité. 


\subsection{L'apport de la philosophie}

Platon et Aristote (427 av. JC), l'identité se définit comme l'égalité de deux éléments ( $\mathrm{X}=$ $\mathrm{X})$, peu de temps après, le principe de l'identité est reconnu par Aristote, comme l'explique Grey (2004): "partie des principes fondamentaux de la pensée métaphysique".

D'autres théories ont étés développés par les philosophes, Notamment, la théorie des indiscernables par Leibnitz; Dodd a développé the identity theory of truth en 1925, the identity theorem in mathematics de Schwarz; en 1970, identity theory of mind développée par Kripke; deux ans plus tard the identity theory of predication de Geacha a émergé, et en 1976 the identity theory in psychology est proposée par R. Carnan. Les trois dernières appartiennent au champ philosophique (Bothamley, 1993).

J. Habermas (1987), le philosophe et le sociologue allemand défend le fait que la conception d'identité permet d'agencer l'identité sociale et l'identité culturelle et il développe ce qu'il nomme "l'action communicationnelle", dans laquelle le "monde des objets" comprend l'architecture, l'urbanisme et le patrimoine.

Habermas, développe le fait que les identités façonnent un sens d'appartenance à une collectivité dont les membres peuvent le dire "catégoriquement".

Si on compare cette approche de l'identité avec celle de Ricoeur (1990), on constate que celle-ci est se focalise plus sur le côté social.

\subsection{L'apport de la psychologie}

En Psychologie, l'Identité sociale (le sentiment ressenti par un individu d'appartenir à un groupe social, et qui le porte à adopter certains comportements typiques). C'est en effet l'ensemble de fait et de droit (date, lieu de naissance, nom, prénom, filiation, etc.) qui permettent d'individualiser quelqu'un. Exemple: vérifier l'identité de quelqu'un.

Selon Erik Erikson l'identité se résume en un sentiment d'harmonie: l'identité de l'individu est le "sentiment subjectif et tonique d'une unité personnelle et d'une continuité temporelle" (1972). D'après Freud l'identité est une construction caractérisée par des discontinuités et des conflits entre différentes instances le Moi, le Ça et le Surmoi. Ces deux conceptions parlent de l'identité comme d'une construction diachronique.

Freud décrit ainsi trois formes d'identification et leur évolution:

a) Le premier est celle de l'enfant qui cherche à reproduire l'image de son père ou de sa mère avant de s'en détacher.

b) La seconde est celle d'une foule où, selon cette analyse, tous les sujets font de leur chef leur "idéal du moi".

c) La troisième est celle qui unit ces sujets entre eux par-delà les différences personnelles.

Cependant, les processus d'identification se repèrent en de multiples phénomènes et pratiques. On parle de "L'identification et socialisation, qui concerne l'enfant par exemple. Ainsi, l'identification est fortement présente dans la socialisation, les apprentissages scolaires ne cessent de provoquer des processus d'identification aux conséquences positives ou négatives" (Dubar, 1991). L'enfant s'identifie au maître, à un groupe de pairs ou / et à un groupe de jeu. L'adolescence est communément marquée par un changement des identifications, parfois difficiles ou traumatisantes.

\subsection{L'apport de la psychanalyse}

Beaucoup de chercheurs (M. Mead, D. Winnicott, M. Kahan, H. Wallon, R. Zazzo, R. Rodríguez, H. Tomé, etc.) s'accordent à dire, que c'est le psychanalyste E. K. Eriksson qui fut l'un des premiers penseurs à donner une définition élaborée et rigoureuse à la notion d'identité dans les années soixante. Selon E. K. Eriksson "le terme identité renvoie à un 
sentiment conscient de spécificité individuelle, tantôt à un effort inconscient tendant à établir la continuité de l'expérience vécue et pour finir la solidarité de l'individu avec les idées d'un groupe".

En analysant sa perception de l'identité on s'aperçoit qu'il existe un paradoxe marqué par le concept de "l'individu" (du latin individuum: corps indivisible); désigne par son origine, d'une part, tout être vu indépendamment comme unique et, d'autre part, un ensemble de traits communs chez les êtres d'un même groupe (espèces, ethnies, genres etc.).

Un débat dialectique français sur l'identité donne lieu à la comparaison entre le "même" et "l'autre". Et c'est le philosophe Ricœur dans sa théorie de la perspective herméneutique du soi, Dans ce sens Ricœur distingue deux aspects majeurs de l'identité; une identité inchangeable qui persiste à travers le temps et qu'il nomme mêmeté, et une identité changeante appelée ipséité "l'identité au sens ipse n'implique pas l'assertion concernant un prétendu noyau non changeant de la personnalité".

Mais cette définition infère à une plus grande complication vue la quantité des combinaisons et éventuels niveaux de similitude-différence engendrant donc une infinité de critères potentiels de singularisation. De ce fait, on peut être semblable par exemple parce qu'on est: humain, jumeau (vrai ou faux), de la même couleur de peau, du même genre, etc. Mais unique par notre caractère, notre façon d'être notre personnalité, etc.

Dans cette même vision, Le concept d'identité désigne conjointement la recherche de l'identique, du semblable et en même temps la quête continue de l'unicité. Ceci impose donc le paradoxe perpétuel celui de la ressemblance et de la différence.

D'autre part, la construction de l'identité vise la conflictualisons de contenus et de niveaux a priori hétérogènes ou contradictoires tels l'individuel et le collectif, le psychique et le social.

L'identité peut en effet, relever d'un processus aussi bien individuel que collectif. A l'échelle de l'individu, l'identité peut être un état résultant, par exemple, d'une assignation administrative: ma carte d'identité établit mon sexe, mon âge, mon domicile, etc.

Dans ce schéma, l'identité peut être assimilée à un concept générique définissant l'état de la personne et de son groupe auquel elle veut se référer puisque cette dernière traduit les comportements individuels et collectifs.

Ainsi, ce processus d'identification consiste, en effet, à reconnaitre ce qui est identique ou semblable et appelle aussi d'autres confrontation et paradoxes entre la réalité et son image, l'identité pour soi et celle pour autrui, l'image de soi et l'image sociale, le passé et le présent. En ce sens, l'identité apparaît comme le résultat d'un processus social car elle se constitue dans des interactions sociales, à partir du regard et de la reconnaissance d'autrui (...) L'identité peut ainsi être définie comme une représentation de soi qui se développe dans la relation à l'autre.

\subsection{L'apport de la sociologie, l'anthropologie et l'ethnologie}

La participation des sociologues et des anthropologues à la question de l'identité est très importante que ce soit dans les anciens ou les nouveaux mouvements sociaux. En effet, le sociologue E. Durkheim (1858-1917) a introduit le concept d'anomie, pour caractériser certains états d'esprits des groupes marginaux et il a baptisé sa théorie; la théorie des conflits des groupes sociaux.

Les sociologues alors, résument la notion de l'identité dans la problématique du rapport entre le collectif et l'individuel. L'identité individuelle est ici le fruit d'un phénomène de socialisation vu par Wagon comme l'outil sociologique le plus pertinent pour décrire le 
processus d'appropriation de l'espace par lequel un individu accède à l'être social, construit les modalités de son "être-ensemble".

L'identité culturelle quant à elle traduit l'ensemble de toutes cultures propres à un groupe ethnique (langue, religion, art, etc.) qui lui confèrent son individualité; sentiment d'appartenance d'un individu à un groupe.

Dans une même logique, Arjun Appadurai s'est par exemple intéressé aux phénomènes d'hybridation ethnique et culturelle dans les conditions techno-politiques de la mondialisation. Dans lequel l'identité occupe une place centrale dans le processus de mondialisation.

L'anthropologie donne une signification assez individuelle par rapport aux autres disciplines. Dans un séminaire sur l'identité organisé dans les années quatre-vingts par LéviStrauss, et André Greren ont définit l'identité comme une composante ayant trois principales caractéristiques; la permanence (en se référents aux points constants et inchangeables) le marquage d'un groupe (ou d'un ensemble d'individus qui permet de dire qu'ils partagent la même identité), l'interprétation (en mettant le point sur la relation existante entre les éléments dans le but d'établir un rapport de similitude entre différents groupes),

Les ethnologues eux, mettent le point sur l'histoire, la religion, le patrimoine, éventuellement les monuments historiques et tout ce qui touche au développement du territoire contemporains M. Auge (2006).

Dans une perspective plus récente sur le débat de l'identité, le sociologue A. Touraine, élargit la recherche et se penche sur l'identité sociale, pour lui il existe trois monde qui virevoltent autours de l'être social: le monde des objets, le monde de la raison et le monde de l'individu lui-même et il le caractérise de dynamique, car il tente par tous les moyens de s'appuyer sur son identité (c'est-à-dire ses racines, ses traditions, sa nation, sa religion, etc. ) dans ce monde en mouvement.

\subsection{L'apport des géographes}

La question de l'identité change de dimension lorsqu'on se situe dans la perspective des géographes. G. Di Méo (2006). Le concept est largement étudiée par les géographes et pour lequel il n'existe pas de consensus, sauf par certains aspects comme: la pluralité d'identités (Monnet \& Capron, 2000), la tendance à passer de l'identité à l'identification.

Les géographes départagent deux concepts, il s'agit de l'identité et de l'identification. En fait, la différence entre les deux concepts n'est pas insignifiante. La notion d'identité a un double sens matériel et immatériel et a besoin d'être soutenue et renforcée, et la démarche de la valorisation de cette identité passe obligatoirement par l'identification; le processus d'identification est permanent, à l'inverse de ce qui se passe avec l'identité qui est perçue comme "une figure essentielle et un peu figée".

Le sentiment identitaire quant à lui, dépend assez souvent de l'espace géographique; on parle alors de l'identité géographique, cette dernière agie au niveau de l'individu, par référence à un espace bien déterminé auquel il est sentimentalement attaché. Lorsque ces sentiments identitaires individuels sont regroupés, ils peuvent donner naissance à des sentiments collectifs d'identité territoriale (Guermond, 2007). La principale particularité disciplinaire de la géographie réside dans sa capacité à appréhender le concept d'identité dans sa dimension spatiale. Certains géographes se sont ainsi penchés sur l'aspect multiscalaire de l'identité, en s'intéressant aux multiples relations existant entre les différentes échelles identitaires, au niveau de l'individu, de la collectivité ou encore de l'espace mondial.

D'autre part, les géographes stipulent que la question de l'identité est intimement liée au territoire qui lui fournit le ciment efficace, à la fois matériel et symbolique. Di Méo définit 
l'identité comme "une construction permanente et collective basée sur le partage des valeurs et de traits culturels et par l'appropriation d'un territoire commun qui est généralement la ville tenant à la forte densité humaine et mémorielle de cet espace".

\subsection{L'apport des urbanistes}

En langue française, rare sont les dictionnaires d'urbanisme qui incluent le concept d'identité même le dictionnaire d'urbanisme de référence (Merlin\&Choay, 2005) ne contient aucune définition du terme identité. Nous trouvons néanmoins la définition du terme" image de ville" qui semble converger dans nombreux éléments avec l'identité, particulièrement en ce qui concerne la représentation sociale de la ville ainsi que la valeur sociale.

Le seul ouvrage dans lequel on a cité et essayé d'éclairer le concept est "la cille et l'urbain" (Pumain et al. 2006).l'auteur affirme le fait que le concept a une double direction; le même et l'autre, et donc il reconnait directement l'ambiguïté du concept, Par contre, une chose est sûre; l'identité doit obéir aux principes de la cohésion et de la résonance. Les villes sont alors" des entités collectives aspirant à se doter d'un véritable principe de reconnaissance et de cohésion. Ce travail repose sur des services de communication, par le biais de logos, de slogans, manifestations culturelles, commémorations historiques, drapeaux et armoiries, etc., mais la création d'une identité reste "le croisement aléatoire d'une volonté politique et d'une reconnaissance de la validité des affirmations exprimées dans l'identité proposée" (Pumain et al. 2006).

\section{VERS UNE TYPOLOGIE DE L'IDENTITÉ TERRITORIALE}

L'identité est une construction sociale et culturelle (Di Méo, 2006), elle est le résultat d'une interaction active entre des facteurs psychologiques et sociaux. Dans cette veine, l'identité n'est pas unique ni figée. De plus, on est de plus en plus mobiles, pluri-territoires et donc pluri-identitaires. Dans ce qui suit nous proposons une typologie des identités.

\subsection{L'identité personnelle}

C'est ce qui permet à travers le temps et l'espace à l'individu de rester lui-même dans un groupe social donné et dans ses relations avec l'autre. Pour Di Méo (2006) il existe six caractères qui déterminent la construction de l'identité personnelle; la continuité, la cohérence, l'unicité (originalité), la conception de la diversité intérieure, la réalisation de soi par l'action et en fin, l'estime de soi. Cette identité est différente d'une personne à une autre, et elle se décline selon les groupes. Cette identité personnelle se transforme du singulier au pluriel et peut devenir un puissant outil politique Di Méo (2006).

\subsection{L'identité géographique: régionale ou nationale}

L'identité dépend, selon cette vision, de l'appartenance sociale et surtout géographique: Galland (1997a) met l'accent sur "le retour de l'acteur" sur la scène sociologique pour chercher sa distinction. Le processus d'identification collective porte en lui les spécificités de chaque région, ainsi le chercheur définit l'identité comme un processus que les groupes sociaux se font des villes dans le temps (passé, présent et à venir) et "dans l'histoire" (Galland, 1997b). Il se concentre par la suite sur le moment où l'on voit la collectivité urbaine comme un acteur socio-économique soucieux de l'instauration de la cohésion sociale, l'idée de "l'identité urbaine" devient alors opérationnelle. De sa part, Mangin (2009) définit l'identité comme une création collective et culturelle perpétuellement en devenir. 
Selon l'auteur l'identité régionale est "plurielle", et les régions n'ont pas toutes des spécificités culturelles fortes, lorsque ce cas se présente le territoire se crée une identité qui devient petit à petit un élément clé de son développement. Subséquemment, la relation et les enjeux des acteurs sur le territoire sont primordiaux pour l'intérêt des pouvoirs publics locaux et régionaux qui se lancent dans des actions pour créer ou défendre une identité Mangin (2009).

\subsection{L'identité valorisante et l'identité dévalorisante}

Les sociologues P. Bourdieu et P. Centilivres, M. Bassand (1991) développent le concept du" stock" d'identités, ce dernier dépend des emblèmes bâtit qui peuvent être valorisantes (positives) ou dévalorisantes (négatives), ainsi, les signes et les symboles socio-culturelles ou historiques choisis sont très délicats du fait que c'est eux qui représentent la région et qui nous permettent de se présenter et de se différencier de l'autre.

Par ailleurs, les travaux de M. Bassand (1991), consistent à proposer une typologie des acteurs régionaux de l'identité. Il fonde son étude sur les oppositions entre "stéréotype" et "stigmate" développées plutôt par le sociologue nord-américain E. Goman (1973). De ce fait, la présence de l'un ou l'autre sur le territoire implique la négation et la dévalorisation du territoire (ici on parle de la région) et donc des individus la constituant (Ces travaux ont été faits principalement à l'échelle régionale du territoire suisse). Pour ce sociologue, l'identité est "un phénomène à la fois individuel et collectif supposant acquisitions et entretien, et devant permettre à l'individu d'effectuer une distinction psychosociale permanente. Elle ne fait que rarement l'unanimité; car ce qui est emblème pour certains est stigmate pour d'autres" (Bassand, Rossel, \& Hainard, 1993). Cette nouvelle définition nous permet donc de dégager deux autre caractéristiques de l'identité; le caractère subjectif et le caractère temporel. Néanmoins, Bassand (1991) pense que la problématique identitaire est "l'exclusivité" des régions périphériques et non pas des villes centrales. Les stéréotypes locaux sont le résultat d'un passé significatif transformée dans le présente et dans le future en une stratégie basée sur la nostalgie pouvant facilement constituer un produit touristique.

Par ailleurs, l'identité peut aussi dans certains cas favoriser les échanges interculturels lors des voyages, comme elle peut dans le cas d'immigration garantir l'intégration des individus au sein des sociétés d'accueil.

De surcroît, elle permet de concilier les différences et crée un sentiment qui nous permet de ne pas se sentir contradictoires. Bien que l'identité est renfermée à cause du stéréotype et des perceptions parfois très loin de la réalité, l'identité n'est statique ni figée, elle n'est pas non plus crispée sur des origines éternelles, mais elle est évolutive et en perpétuel croissance en croissance. En revanche, l'identité est très ouverte et tolérante, elle permet d'accepter et de dialoguer avec l'autre, c'est-à-dire le reconnaître malgré les tensions résultant de la confrontation des diversités.

\subsection{L'identité ouverte et l'identité fermée}

Toute identité et/ou processus d'identification renvoie aux situations de métissages et d'hybridations culturelles (Castells, 1999).

Les identités spatiales fermées selon les géographes peuvent fonctionner en étant isolés géographiquement et politiquement (Monnet \& Capron, 2000). Cependant, cette acceptation ne pourrait aboutir qu'à 'l'appauvrissement, qu'à la formation de ghettos, qu'à la ségrégation et l'asservissement. A contrario, les identités ouvertes portent l'innovation et le progrès sociaux, la démocratie, la durabilité sociale au sens fort du terme” (Di Méo, 2007). 
L'économiste Clark Greg (OCDE, 2009) reprend la même idée et développe cette même hypothèse dans sa stratégie sur l'ouverture des métropoles au niveau national et international.

\subsection{L'identité individuelle et l'identité collective}

Paasi (2002), plonge dans la recherche de ce lien entre la dimension personnelle et la dimension collective de l'identité et note que "les liens entre les dimensions personnelles et collectives de l'identité demeurent confus". Les identifications régionales sont très diverses et multiples et la transformation de l'identité territoriale ponctuelle en identité collective est un fait. Cette identité est en général basée sur des traits et des déterminants culturels fondés spécialement sur les valeurs. Ces derniers sont selon G. Di Méo "inculqués ou rappelées, ressassées en permanence par l'œuvre inlassable des appareils idéologiques" (Raffestin, 1980) complète l'idée en notant que "tout objet peut endosser une fonction patrimoniale, et tout espace peut devenir territoire, à la condition qu'ils soient, l'un et l'autre pris dans un rapport social de communication". Néanmoins, les origines, l'histoire, la langue, l'espace et même les émotions ne sont pas suffisants pour créer le sentiment d'appartenance et le besoin de vivre ensemble. En fait, l'adoption d'un projet pour l'avenir comme un projet de culture démocratique peut participer à l'émergence d'une identité politique partagée Lacroix (2004).

\section{L'IDENTITÉ TERRITORIALE: PIERRE ANGULAIRE DE L'ATTRACTIVITÉ}

L'identité territoriale est souvent un sujet problématique, polémique, voire explosif, car elle connote, et instrumentalise politiquement et idéologiquement.

A première vue, le processus d'identification renvoie à la dimension spatiale et nous pouvons distinguer le territoire selon la taille (régionale, communale, etc.). Conséquemment, l'identité est représentée "par des mécanismes lies à l'espace" (Di Méo, 2006). Ce chercheur géographes évoque aussi à une échelle plus totale, pourquoi les identités" différentielles et territoriales" s'appuient sur des facteurs culturels, religieux et paysagers. Il existe donc une fabrique d'identités classées selon" une hiérarchisation des appartenances" au territoire.

L'ethnologue M. Auge (2006) de sa part, défend l'idée qui stipule que c'est le langage qui se trouve au centre du problème de l'identité et le territoire. Pour lui, les problématiques liées à ces deux notions sont au cœur de nos malaises. Pour lui, le territoire est "l'espace qui se définit par le partage d'un langage", selon Auge (2006), les non-lieux sont en même temps des lieux sans identité.

L'identité territoriale est définie par le temps; elle est marquée par des ancrages mémoriels sollicitant une présence sur le long terme et mobilisant des marqueurs historiques ou spatiaux aux changements assez lents (Candau, 1998; Marié, 1982). Elle est manifestée comme une sorte d'inertie conduisant à associer l'identité à la résistance au conservatisme à la stabilité, au non modernité et au déni du changement.

Cette représentation est réductrice du sens large de l'identité qui est assimilée au “... processus de construction de sens à partir d'un attribut culturel, ou d'un ensemble cohérent d'attributs culturels, qui reçoit priorité sur toutes les autres sources". (Castells, 1997).

Dans l'identité territoriale, c'est le territoire en tant qu'objet spatial porteur de signes culturels et doté de sens qui alimente la construction du sens, du sujet ou de la collectivité. La référence à un espace fonde la conscience d'une singularité collective; la singularité sociale et culturelle s'alimentant de la singularité de l'espace qu'elle conduit à produire. Ainsi 
L'identité territoriale est assimilée aux procédures de la symbolisation, de l'inscription dans l'espace des structures fondatrices de la vie collective: mythes et histoire, autant que centre et limites (Segaud, 1983; Bonnemaison, 2000).

L'identité des territoires est donc définie dans une singularité qui lui confère un statut d'objet propre et d'objet spatial. En tant que telle, elle relève d'abord d'un acte de catégorisation sociale (Dubar, 1998), permettant de distinguer et de différencier.

En reprenant une définition de Mucchieli (1986), deux types de caractérisation nous semblent pouvoir être distingués, l'un positiviste, l'autre existentialiste. La caractérisation positiviste ressortit de la spécificité. Elle fait valoir des données qui distinguent une région parmi d'autres sur des critères mesurables ou vérifiables.

Dans la même perspective, les tenants de ces théories perçoivent l'identité territoriale comme modalité de l'identité collective. La construction identitaire est donc un processus social qui résulte des relations avec autrui (Mead, 1963). Ce sont les relations entre les individus d'un territoire qui permettent de construire des représentations communes, révélant les connexions cognitives entre individus à l'origine de l'identité territorial (Dutton et al., 1994; Monnet, 2000).

De ce fait un territoire en géographie est déterminé par l'appropriation des individus et des collectifs des territoires et des registres essentiellement cognitifs ou symbolique.

Marie-Christine Fourny, définit l'identité territoriale comme étant "modalité à partir de laquelle une société fonde la conscience de sa singularité en la référant à un espace qu'elle institue sien". De ce fait, l'identité territoriale est "à l'origine un sentiment individuel" (Guermond, 2007)

Par conséquent, en tant que concept psychologique projeté aux territoires, l'identité territoriale peut être répartie en deux niveaux, individuel (cognitif, conatif et affectif) et un niveau collectif ce qu'un territoire est et ce qui le différencie des autres. Par extension l'identité comprend trois dimensions: une dimension cognitive, par laquelle les individus connaissent le territoire et ses limites, une dimension affective, qui reflète l'attachement d'un individu à son territoire, et une dimension conative, liée à la mobilisation de l'individu pour une action collective en faveur de son territoire (Keating, 1998). Elle est dans ce cas paradoxale dans le sens où elle résulte de caractéristiques individuelles et singulières (Dubar et Tripier, 2009).

Le rapport des individus au territoire apparaît dans les définitions les plus basics du territoire notamment celle de Larousse qui le détermine en tant que domaine qu'une personne s'approprie, où elle tente d'imposer ou de maintenir son autorité, ses prérogatives.

Les sociologues ont été parmi les chercheurs qui se sont le plus intéressés à cette relation entre identité et territoire, selon eux pour qu'un territoire existe il faut que l'humain tisse des relations avec ce dernier. Pareillement selon Di Méo (1998) "l'individu socialisé créé un lien avec la terre qu'il pratique". Conséquemment l'humain développe un sentiment d'appartenance à ce territoire. Dans la même mesure, Jodelet témoigne que ce sont "les représentations sociales qui modèlent le territoire".

Dans une autre configuration, le psychologue Piere Tape affirme que l'identité c'est “... ce par quoi je me définis et me connais, ce par quoi je me sens accepté et reconnu comme tel par autrui'. C'est-à-dire que c'est l'ensemble des représentations qui permettent à la personne d'être elle-même et différente des autres en même temps. Pareillement, les anthropologues pensent que l'identité du territoire est l'ensemble des représentations qui permettent d'identifier un territoire. De ce fait il existe un lien fort entre l'identité et le territoire.

Une théorie vient compléter l'analyse, appelée les “Coquilles de l'Hommes". Elle étudie la relation entre l'homme et son espace (son territoire). L'identité de l'homme se constitue du 
quartier, de la ville de la région, en somme de ses repères spatiaux temporels. Parallèlement l'identité d'un territoire se constitue de ses mêmes repères.

De ce fait, l'identité crée le lien entre le territoire et l'individu. En d'autres termes l'individu crée l'identité du territoire et vice versa.

\section{CONCLUSION}

La réflexion et les recherches autours de la notion de l'identité est transdisciplinaire et chaque discipline partant de la sociologie jusqu'aux sciences sociales oriente et interprète la définition comme une représentation, une démarche, un processus, une construction ou un état selon sa perception et ses objectifs.

L'identité est une notion sociale et peu scientifique dans le sens où il n'est pas possible de mesurer le degré d'adhésion d'une population à cette identité, qui est fluctuant en fonction du contexte historique et politique. Elle est influencée par des contrastes historiques, sociaux, politiques et même économiques.

L'ambiguïté de ce concept réside dans le fait qu'il est un phénomène social, intellectuel et sentimental aux contours flous. Au niveau territorial il est représenté sous forme de territoires précis et délimités dans le temps et l'espace par des frontières et limites représenté sous forme de découpage politique, une carte figée délimitant les territoires et non les populations. Alors que l'identité constituée commencement et le commandement de toute démarche d'attractivité territoriale durable et le cas des grand territoires qui ont réussi leurs stratégies d'attractivité en est le témoin: Lyon, Bretagne, etc. Dont le portrait identitaire était la locomotive de leur marketing territorial.

\section{BIBLIOGRAFÍA}

Abakouy, M. y Alaoui, Y. (2015) "Modélisation de la démarche marketing territorial", Revue de mangement, 3: 42-60.

Appadurai, A. (1996) Modernity at Large: Cultural Dimensions of Globalization. Minneapolis: Univesity of Minnesota Press.

Auge, M. (2006) L'anthropologie. Paris: Presse Universitaire de France.

Bassand, M. (1991) Identité et développement régional, Conseil de l'Europe, projet culture et région. Berne: Peter Lang.

Bassand, M.; Rossel, P. y Hainard, F. (1993) Animations et identités. Gestion territorialisée des crises. Lausanne: L'âge d'homme.

Bonnemaison, J. (1995) “Le territoire, nouveau paradigme de la géographie humaine?", Acte du colloque, Le territoire, lien ou frontière?, 2-4 octobre, Paris-Sorbonne: pp.1-4.

Bothamley, J. (1993) "Identity" Dictionary of theories. Gale research International Ltd: United Kingdom, p. 326.

Bourdieu, P. (1982a) Ce que parler veut dire: l'économie des échanges linguistiques. Paris: Fayard.

Bourdieu, P. (1982b) Leçon sur la leçon. Paris: Les Editions de Minuit.

Candau, J. (1998) Mémoire et identité, Collection Sociologie d'aujourd'hui. Paris: Presses Universitaires de France.

Castells, M. (1999) Le pouvoir de l'identité. Paris: Fayard.

Debarbieux, B. (2006) Prendre position: réflexions sur les ressources et les limites de la notion d'identité en géographie. Paris: Fayard.

Di Méo, G. (1998) Géographie sociale et territoires. Paris: Nathan. 
Di Méo, G. (2006) "Le rapport identité/espace. Éléments conceptuels et épistémologiques”, en P. Grandjean Construction identitaire et espace. Paris: Le Harmattan: pp.19-39.

Dubar, C. (1991) La socialisation. Construction des identités sociales et professionnelles. Paris: A. Colin.

Dubar, C. (1996) "La sociologie du travail face à la qualification et à la compétence", en Sociologie du travail, 2: 179-192.

Dubar, C. (1998) "Réflexions sociologiques sur la notion d'insertion", en B. Charlot y D. Glasmann (dirs.) Les jeunes, l'insertion, l'emploi. Paris: PUF.

Dubar, C. y Tripier, P. (2009) Sociologie des professions. Paris: Armand Colin.

Dubois, J.; Mitterand, H. y Dauzat, A. (1998) Dictionnaire étymologique et historique du français. Paris: Larousse.

Durkheim, M. (1900) "La Sociologie et son domaine scientifique", Version francophone de "La sociologia e il suo domino scientifico", in Rivista italiana di sociologia, 4, 1900; pp.127-148.

Dutton, J. E.; Dukerich, J. M. y Harquail, C. V. (1994) "Organizational images and member identification". Administrative science quarterly, 39 (2): 239-263.

Erikson, E. (1978) Adolescence et crise. La quête de l'identité. Paris: Poche.

Fourny, M.C. (2005) "Identités et dynamiques territoriales. Coopération, différentiation, temporalités", en Mémoire d'habilitation à diriger des recherches, Grenoble, Université Joseph Fournier, p. 122.

Freud, S. (2013) L'inconscient. Paris: Dominique Renauld.

Galland, O. (1997a) "Parler en famille: les échanges entre parents et enfants", Économie et Statistique, 304-305 (4/5): 163-177.

Galland, O. (1997b) "Leaving home and Family Relations in France", Journal of Family Issues, 18 (6): 645-670.

Granjon, F. (2002) Note de lecture: A. Appadurai (2001) Après le colonialisme. Paris: Payot, en Commposite.org, accisible en https://goo.gl/em9zCT, (consulté le 11 janvier 2016).

Guermond, Y. (2007) "L'identité territoriale: l'ambiguïté d'un concept géographique", L'Espace géographique, 4: 290-297.

Habermas, J. (1987). Théorie de l'agir communicationnel, Tome II: Pour une critique de la raison fonctionnaliste, Paris: Broché.

Jodelet, D. (1991) Les représentations sociales, Paris: UF.

Keating, M. (1998) The new regionalism in Western Europe. Cheltenham: Edward Elgar.

Lacroix, J. (2004) "Pour un patriotisme constitutionnel européen", en P. Savidan (dir.) La République ou l'Europe? Paris: Le Livre de Poche.

Levi-Strauss, C. (1979) Résumé des cours et travaux (1978-1979). Paris: Annuaire du Collège de France.

Marié, M. (1982) Un territoire sans nom, pour une approche des sociétés locales. Paris: Librairie des Méridiens.

Mead, G. H. (1963) L'Esprit, le soi et la société. Paris: PUF.

Merlin, P. y Choay, F. (2005) Dictionnaire d'urbanisme et de l'aménagement du territoire. Paris: PUF.

Monnet, J. (2000) "Les dimensions symboliques de la centralité", Cahiers de Géographie du Québec, 44 (123): 399-418, DOI: 10.7202/022927ar

Noisette, P. y Vallerugo F. (2010) Un monde de villes. Le marketing des territoires durables. ClermontFerrand: Editions de l'Aube.

Paasi, A. (2002) "Bounded spaces in the mobile world: deconstructing regional identity", TESG 93 (05): 37-148.

Pumain, D.; Paquot T. y Kleinschmager, R. (2006) Dictionnaire La ville et l'urbain. Paris: EconomicaAnthropos.

Raffestin, C. (1980) “Réinventer l'hospitalité”. Communications, 65: 165-177.

Rey, V. y Van Den, C. (1998) "Langue et identité en situation migratoire; identité ethnique", Identité linguistique-A chacun son balbara, 6 (11): 129-134.

Segaud, M. y Levy, P. (1983) Anthropologie de l'espace. Paris: Centre Pompidou.

Tape, P. (1980) Identités collectives et changements sociaux. Paris: Privat.

Touraine, A. (1996) Identité et modernité. Québec: les Presses de l'Université Lval. 


\section{Breve currículo:}

\section{Yasmine Alaoui}

Doctorante $\mathrm{Au}$ Groupe De Recherche En Entrepreneuriat, Finance, Audit Et Marketing Ecole Nationale De Commerce Et De Gestion De Tanger lauréate de l'Ecole Nationale de Commerce et de gestion de Tanger, Option Marketing et action commerciale (Université Abdelmalek Essaâdi). Lauréate de l'Institut de Management Public et Gouvernance territoriale. Option Marketing territorial (Aix-Marseille Université). Enseignante à ESCA école de management, Casablanca. Maroc.

\section{Mostafa Abakouy}

Enseignant chercheur à l'ENCG de Tanger (Université Abdelmalek Essaâdi). Membre du Conseil d'Etablissement et responsable de la Filière" Commerce" (ENCG - Tanger). Spécialisé en Management International des PME et Marketing Territorial. Coordinateur de l'Equipe de Recherche" Marketing et Commerce International" et membre fondateur du Groupe de Recherche en Entrepreneuriat, Finance, Audit et Marketing (ENCG - Tanger). Membre fondateur du Centre de Recherche et des Etudes en Economie et Gouvernance Régionales et Urbaines (FSJES - Tanger). Membre du Comité de Rédaction et de Lecture de la Revue Marocaine de Management Logistique et Transport. 